\title{
Adsorción y oxidación de monóxido de carbono en una lamina de $\beta$-grafino: Estudio teórico
}

\author{
Adsorption and oxidation of carbon \\ monoxide in a sheet of $\beta$-Graphyne: \\ Theoretical study
}

\author{
Yesica Celeste Villagrán López ${ }^{1}$, Samanta Magalí Carrión ${ }^{1,2}$, \\ María Beatríz López ${ }^{1}$
}

\footnotetext{
${ }^{1}$ Centro de Investigaciones Fisicoquímicas, Teóricas y Aplicadas, Facultad de Ciencias Exactas y Naturales, Universidad Nacional de Catamarca, Av. Belgrano 300, 4700, Catamarca, Argentina.

${ }^{2}$ Centro de Investigaciones y Transferencia de Catamarca, CONICET, Catamarca, Argentina.

e-mail: celestevillagranlopez@gmail.com
}

\section{RESUMEN}

En este trabajo se presenta un estudio, basado en la Teoría del Funcional de la Densidad (DFT), de la adsorción y oxidación de CO sobre un catalizador libre de platino constituido por una lámina de grafino, como potencial catalizador de celdas de combustible. El Grafino es un nuevo alótropo del carbono, su estructura es bidimensional y está constituido por una monocapa de átomos de carbono con hibridación sp-sp2. Dependiendo de las diferentes formas de insertar el triple enlace carbono-carbono se puede distinguir tres tipos de Grafino: alfa, beta y gamma Grafino.

Se han estudiado las propiedades estructurales, energéticas, electrónicas y reactividad química frente a la adsorción y oxidación de monóxido de carbono de una lámina de $\beta$-Grafino. Se propone una posible ruta de reacción, mediante el mecanismo Eley-Rideal. Los cálculos fueron realizados usando DFT y se utilizó el funcional híbrido B3PW91 y la base 6-31 G para la optimización de los átomos de carbono y oxígeno, el estado de transición se obtuvo a partir del método QST2, además se calcularon las frecuencias vibracionales para confirmar el estado de transición y las coordenadas de reacción intrínsecas (IRC) para obtener el mecanismo de reacción. Todos los cálculos se realizaron según el formalis mo del programa Gaussian09.

Nuestros resultados indican que la coexistencia de carbonos con hibridación sp y sp2 en grafino promueve que átomos de carbono perciban características electropositivas lo que favorece la disociación de oxígeno y posterior oxidación de CO. El estudio de la reacción de oxidación a través del mecanismo Eley- Rideal demuestra que es un proceso concertado, exotérmico y espontaneo.

Palabras clave: $\beta$-grafino, teoría del funcional de la densidad, adsorción y oxidación de CO.

\section{ABSTRACT}

This work presents a study, based on the Density functional theory (DFT), of the adsorption and oxidation of $\mathrm{CO}$ on a free platinum catalyst constituted from a sheet of Graphyne.

Graphyne is a new allotrope of carbon, its structure is two-dimensional and is constituted by a monolayer of carbon atoms with sp-sp2 hybridization. Depending on the different ways to insert the carbon-carbon triple bond, three types of Graphyne can be distinguished: alpha, beta and gamma Graphyne.

The structural, energetic and electronic properties have been studied, as well as the chemical reactivity to the adsorption and oxidation of carbon monoxide of a $\beta$-graphyne sheet. Therefore we propose a possible reaction route, using the Eley-Rideal mechanism. The calculations were performed using DFT, the functional hybrid B3PW91 and a 6-31 G basis for optimization of oxygen and carbon atoms was used. The transition state was obtained from the QST2 method, furthermore, vibrational frequencies were calculated to confirm the transition state and intrinsic reaction coordinates (IRC) to obtain the reaction mechanism. All calculations are performed according to the formalism of Gaussian09 program.

Our results indicate that the coexistence of carbon with $\mathrm{sp}$ and $\mathrm{sp} 2$ hybridization in grafino promotes carbon atoms to perceive electropositive characteristics which favors the dissociation of oxygen and subsequent oxi- 
dation of CO. The study of the oxidation reaction through the Eley-Rideal mechanism shows that it is a concerted, exothermic and spontaneous process.

Keywords: $\beta$-graphyne, Density functional theory, $\mathrm{CO}$ adsorption and oxidation

\section{INTRODUCCIÓN}

Una celda de combustible es un dispositivo electroquímico que transforma en energía eléctrica la energía química de la reacción entre un combustible (hidrógeno, hidrocarburos, alcoholes) y un oxidante (por ejemplo, oxígeno del aire o puro), sin ningún ciclo de combustión intermedio [1].

Las investigaciones más recientes se han centrado en celdas de baja temperatura, en particular las de de intercambio protónico que operan con hidrógeno (PEMFC) y las que operan con metanol (DMFC), ya que estas celdas son las que mejor se adaptan a las aplicaciones en el área automotriz, para la generación de energía en áreas residenciales y, en general, a dispositivos móviles o estacionarios de pequeño tamaño.

Uno de los principales problemas de las celdas de combustible de baja temperatura, es la baja eficiencia de los catalizadores anódico y catódico, conformados usualmente por platino, debido al envenenamiento por $\mathrm{CO}$ y otros intermediarios producidos durante la oxidación [2]. El problema del envenenamiento por CO es especialmente grave en las celdas DMFC dado que el monóxido de carbono está siempre presente en cantidades críticas como un intermediario en la reacción de oxidación de metanol.

En la búsqueda de reducir los costos y mejorar la eficiencia de los catalizadores se han propuesto catalizadores que no contengan platino o con un bajo contenido de éste.

Los materiales de carbono, tales como Nanotubos de carbono, Nanofibras de carbono y Grafeno, son objeto de especial atención como catalizadores libres de platinol [3-7].

El Grafino es un nuevo alótropo del carbono, de estructura bidimensional y está constituido por una monocapa de átomos de carbono con hibridación sp-sp2 [8].

El Grafino fue sugerido por primera vez por Baughman y col. [9] y lo construyeron insertando el enlace acetilénico ( $\mathrm{C} \equiv \mathrm{C}$-) entre dos carbonos unidos en Grafeno. Dependiendo de las diferentes formas de insertar el enlace $-\mathrm{C} \equiv \mathrm{C}$ - se puede distinguir tres tipos de Grafino: $\alpha, \beta$ y $\gamma$-Grafino.

En este trabajo se presenta un estudio, basado en la Teoría del Funcional de la Densidad (DFT), de la adsorción y oxidación de CO sobre un catalizador libre de platino constituido por una lámina de $\beta$-grafino y su análisis termodinámico, además del estudio de las propiedades estructurales, electrónicas y energéticas de $\beta$-grafino. Para llevar a cabo lo propuesto se utilizó el software Gaussian 09[10], utilizando el método B3PW91 y la base 6-31G para la adsorción y el estado de transición. Para la visualización de los resultados se ha empleado la interfaz gráfica GAUSSVIEW 5.0.[11]

\section{MATERIALES Y MÉTODOS}

Todos los cálculos se realizaron usando la Teoría del Funcional de la Densidad (DFT) bajo el formalismo del programa Gaussian 09. Las geometrías de reactivos, estados de transición (ETs) y productos, fueron optimizadas con el funcional híbrido de tres parámetros de Becke 3 para el intercambio y el funcional de correlación Perdew-Wang 91 (B3PW91) [12,13], usando el conjunto base estándar 6-31G. la estructura del ET se localizo utilizando el método QST2, el cual emplea la aproximación de tránsito sincrónico cuadrático para acercarse a la región cuadrática del estado de transición y posteriormente a través del algoritmo cuasiNewton finaliza la optimización.La visualización de los resultados se ha realizado con Gaussview 5.0

En este trabajo se han determinado parámetros estructurales, energéticos y electrónicos de una lámina de $\beta$-grafino y se ha analizado también su reactividad química frente al monóxido de carbono (CO) mediante el cálculo de indicadores de reactividad globales, tales como Gap de energía $(\Delta \mathrm{E})$, potencial químico $(\mu)$, dureza química $(\eta)$ e índice de electrofílicidad $(\omega)$ (Ecuaciones 1 a 4). Una descripción detallada de la metodología para obtener los descriptores de reactividad a través de la Teoría del Funcional de la Densidad puede ser consultada en una publicación de nuestro grupo de trabajo[14]. Se propone además una ruta de adsorción y oxidación de monóxido de carbono en una lámina de $\beta$-grafino a través del mecanismo Eley-Ridley. En este mecanismo[15], sólo una de las moléculas se adsorbe y la otra reacciona directamente desde la fase de gas sin adsorberse.

$$
\begin{aligned}
& \mathrm{A}(\mathrm{g})+\mathrm{S}(\mathrm{s}) \leftrightarrow \mathrm{AS}(\mathrm{s}) \\
& \mathrm{AS}(\mathrm{s})+\mathrm{B}(\mathrm{g}) \rightarrow \text { Productos }
\end{aligned}
$$


La determinación del sitio de adsorción de O se realizó mediante la energía de adsorción (Ecuación 5)y se localizó el estado de transición usando el método QST2 [16], que emplea la aproximación de tránsito sincrónico cuadrático para acercarse a la región cuadrática del estado de transición y posteriormente a través del algoritmo cuasi-Newton finaliza la optimización. Se calcularon también frecuencias vibracionales para verificar la naturaleza de los puntos estacionarios como mínimos o estados de transición de primer orden al presentar solo una frecuencia imaginaria. Asimismo para el estado de transición se realizaron cálculos de "Coordenada Intrínseca de Reacción” (CIR) para verificar que dichos estados de transición localizados conectaban con los correspondientes puntos mínimos estacionarios asociados a reactantes y productos [17-18].

En cuanto al análisis termoquímico, las entalpías y energías libres de Gibbs de activación y de reacción se calcularon teniendo en cuenta las sumas y diferencias apropiadas en cada caso. El paquete Gaussian 09 proporciona las sumas de la energía electrónica y la corrección térmica a la entalpia, así como la corrección térmica a la energía libre de Gibbs, de modo que, realizando la diferencia de estos valores para reactivos, estados de transición y productos se pueden obtener dichos parámetros termodinámicos. Así por ejemplo, mediante las ecuaciones 6 y 7,podemos calcular la entalpía de activación y de reacción respectivamente mientras que con las ecuaciones 8 y 9 las energías libres de Gibbs de activación y de reacción pueden ser calculadas.

$$
\begin{aligned}
& \Delta E=\varepsilon_{\text {LUMO }}-\varepsilon_{\text {HOMO }} \\
& \mu=\left(\varepsilon_{\text {LUMO }}+\varepsilon_{\text {HOMO }}\right) / 2 \\
& \eta=\left(\varepsilon_{\text {LUMO }}-\varepsilon_{\text {HOMO }}\right) / 2 \\
& \omega=\frac{\mu^{2}}{2 \eta} \\
& E_{\text {adi }}=E_{\text {grafino-O }}-E_{\text {grafino }}-E_{O_{\text {[ }}} \\
& \Delta H^{\ddagger}=\left(\varepsilon_{O}+H_{C o r r}\right)_{E T}-\sum\left(\varepsilon_{O}+H_{\text {Corr }}\right)_{\text {Reactivos }} \\
& \Delta H^{\circ}=\left(\varepsilon_{O}+H_{\text {Corr }}\right)_{\text {Productos }}-\sum\left(\varepsilon_{O}+H_{\text {Corr }}\right)_{\text {Reactivos }} \\
& \Delta G^{\ddagger}=\left(\varepsilon_{O}+G_{C o r r}\right)_{E T}-\sum\left(\varepsilon_{O}+G_{C o r r}\right)_{\operatorname{Re} a c t i v o s} \\
& \Delta G^{\circ}=\left(\varepsilon_{O}+G_{\text {Corr }}\right)_{\text {Productos }}-\sum\left(\varepsilon_{O}+G_{\text {Corr }}\right)_{\text {Reactivos }}
\end{aligned}
$$

\section{RESULTADOS Y DISCUSIÓN}

\subsection{Análisis energético y de reactividad de la lámina de $\beta$-grafino.}

En La Tabla 1se exponen los resultados en valor absoluto de la energía total Et para la lámina de $\beta$-grafino expresada en unidades de eV. Con la información de la energía de los orbitales de frontera HOMO (Highest Occupied Molecular Orbital) y LUMO (Lowest Unoccupied Molecular Orbital) se calculó la diferencia entre ellos denominada Gap de energía $(\Delta \mathrm{E})$, ésta es una magnitud que permite realizar una primera predicción de la reactividad del sistema en estudio. Además se detallan los siguientes indicadores de reactividad global: potencial químico $(\mu)$, dureza química $(\eta)$ e índice de electrofílicidad $(\omega)$.

El potencial químico está relacionado con la tendencia de un sistema a transferir electrones. En consecuencia, cuanto mayor sea $\mu$, más facilidad de ceder electrones tendrá el sistema. La dureza química $(\eta)$ es una magnitud que mide la resistencia que impone un sistema a modificar su distribución electrónica. Cuanto menor sea se espera mayor reactividad. El índice de electrofílicidad $(\omega)$ mide la tendencia de captar electrones, de manera que indica cuan electrofílico será el sistema. Su aumento favorecerá a la actividad catalítica.

La Figura 1 muestra la estructura de la lamina de $\beta$-grafino, las distancias interatómicas y la hibridación de 
los diferentes átomos. En la Tabla 1 se resumen los parámetros energéticos y los indicadores de reactividad para la lamina de $\beta$-grafino

Tabla 1: Análisis energético y de reactividad global para $\beta$-grafino.

\begin{tabular}{l|l|l|l|l|l|l|l}
\hline SISTEMA & $\mathrm{E}_{\mathrm{T}}[\mathrm{eV}]$ & $\mathrm{E}_{\text {номо }}[\mathrm{eV}]$ & $\mathrm{E}_{\mathrm{Lumo}}[\mathrm{eV}]$ & $\boldsymbol{\Delta} \mathrm{E}[\mathrm{eV}]$ & $\boldsymbol{\mu}[\mathrm{eV}]$ & $\boldsymbol{\eta}[\mathrm{eV}]$ & $\boldsymbol{\omega}[\mathrm{eV}]$ \\
\hline B-grafino & 74535,55 & $-5,707$ & $-5,347$ & 0,36 & $-5,527$ & 0,18 & 84.855 \\
\hline
\end{tabular}
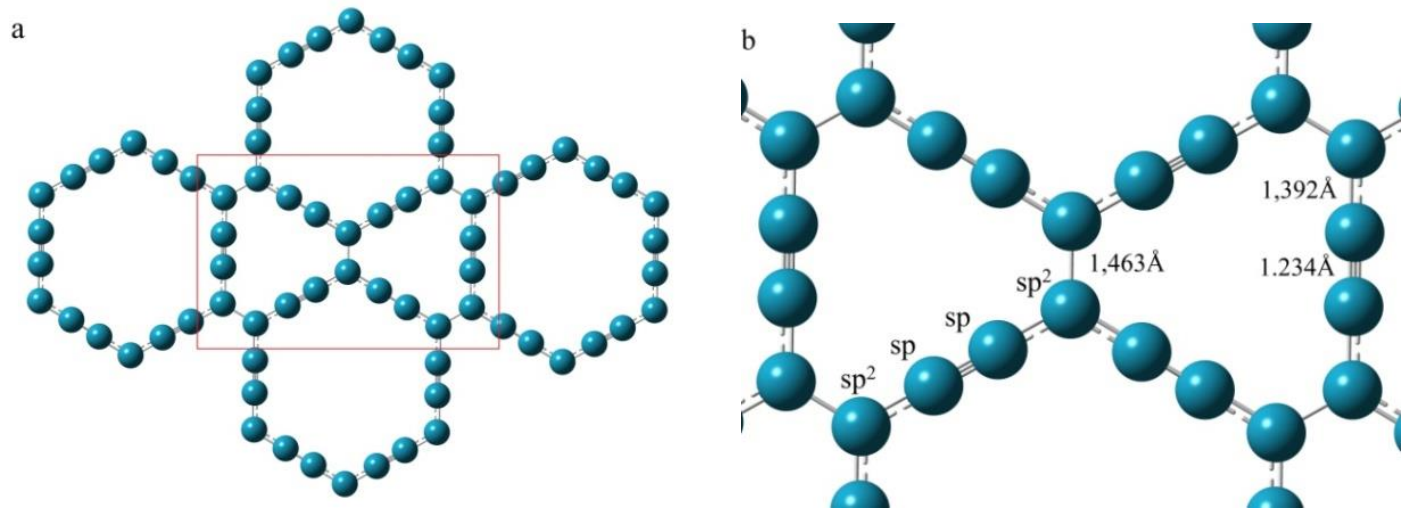

Figura 1: a) estructura de $\beta$-grafino. b) estados de hibridación de los átomos de carbono y longitudes de enlace.

Se calcularon las densidades de carga para cada átomo de carbono a partir del análisis de cargas de Mulliken. El color rojo indica carga electronegativa, mientras que el verde carga electropositiva. Cuando las cargas electropositivas son muy pequeñas, la coloración aumenta el contraste llegando a negra. En la figura 2a se puede apreciar que los átomos de carbono con hibridación sp son los que presentan densidades de carga electronegativas, mientras que los átomos vecinos a los enlaces - $\mathrm{C} \equiv \mathrm{C}-$, con hibridación $\mathrm{sp}^{2}$, presentan densidades de carga positivas. En la figura $2 \mathrm{~b}$ se observa cargas altamente negativas que adquieren valores entre $-0,180 \mathrm{a}$ $-0,220$ u. a. en el centro de la lámina, donde se realizaron las adsorciones tanto de oxígeno como de monóxido de carbono. Mientras que los átomos que tienen cargas electropositivas de 0,120 u.a. en adelante están representados por una fuerte coloración verde.

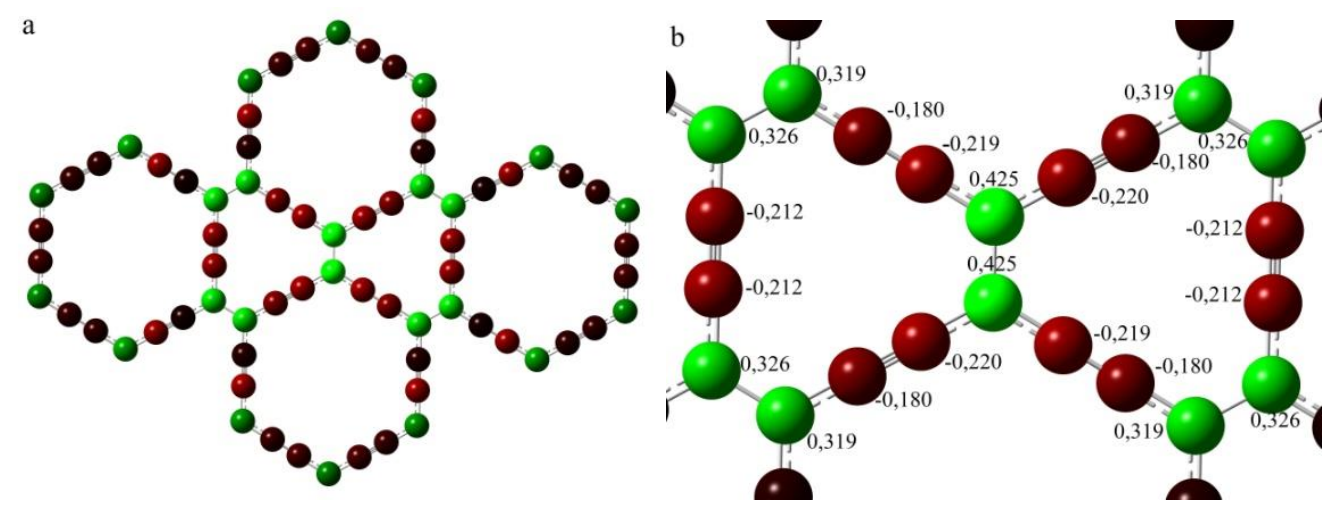

Figura 2: a) Análisis de distribución de cargas para la lámina de $\beta$-grafino, el color rojo y el verde corresponden a cargas negativas y positivas respectivamente. b) Valores de densidad de carga para átomos en los sitios de adsorción.

\subsection{Adsorción de Oxígeno y Monóxido de Carbono}

Se adsorbieron átomos de Oxígeno y moléculas de monóxido de carbono en diferentes sitios de la lámina de $\beta$-grafino. Debido a las diferencias en las energías de adsorción de los diferentes sistemas, se escogió el sis- 
tema GR/O-II para realizar el mecanismo de reacción. Al presentar este sistema una menor energía de adsorciónnos permitió elegir el mecanismo de Eley-Rideal para proponer una posible ruta de reacción. En la Tabla 2 se muestran las energías de adsorción para los diferentes sistemas.

Tabla 2: Energías de adsorción

\begin{tabular}{l|l|l|l|l}
\hline & GR/O I & GR/O II & GR/CO I & GR/CO II \\
\hline$E_{A D}(e V)$ & $-5,038$ & $-5,094$ & $-0,744$ & $-0,643$ \\
\hline
\end{tabular}

\subsection{Oxidación de CO mediante Mecanismo Eley-Rideal}

La oxidación de $\mathrm{CO}$ sobre la lámina de $\beta$-grafino se ha realizado por medio del mecanismo Eley-Rideal, que consistió en la adsorción de un átomo de oxígeno sobre diferentes sitios de la lámina de $\beta$-grafino, y la posterior elección del sistema con menor energía de adsorción. A dicho sistema se le aproximó una molécula de monóxido de carbono en estado gaseoso que sustrajo el átomo de oxigeno produciéndose su oxidación a dióxido de carbono.

Grafino-O $(\mathrm{ad})+\mathrm{CO}(\mathrm{g}) \rightarrow$ Grafino $(\mathrm{s})+\mathrm{CO}_{2}(\mathrm{~g})$

Asimismo, se localizó el estado de transición ET usando el método QST2 y también se calculó la frecuencia vibracional. Para la caracterización del estado de transición localizado se usó el método de la "Coordenada Intrínseca de Reacción" ( IRC, Intrinsec Reaction Coordinate) (Figura 3) para verificar que dicho estado de transición localizado conectaba con los correspondientes reactivos y productos. Se analizaron parámetros termodinámicos para el mecanismo de oxidación propuesto. La Figura 4 muestra el diagrama de energías para la reacción de oxidación con las estructuras de Reactivos, E.T y Productos con sus respectivas entalpías y energías libres de Gibbs, expresadas en unidades atómicas (a.u.). La Tabla 3 brinda los valores obtenidos para las entalpías y energías libres de activación y de reacción, los cuales indican que el mecanismo de oxidación de $\mathrm{CO}$ sobre la lámina de $\beta$-grafino es un proceso exotérmico y espontaneo Además se calculó la frecuencia vibracional, obteniéndose un único valor de frecuencia imaginaria $\left(604,16 \mathrm{im}^{-1}\right)$ que confirma la naturaleza del E.T.

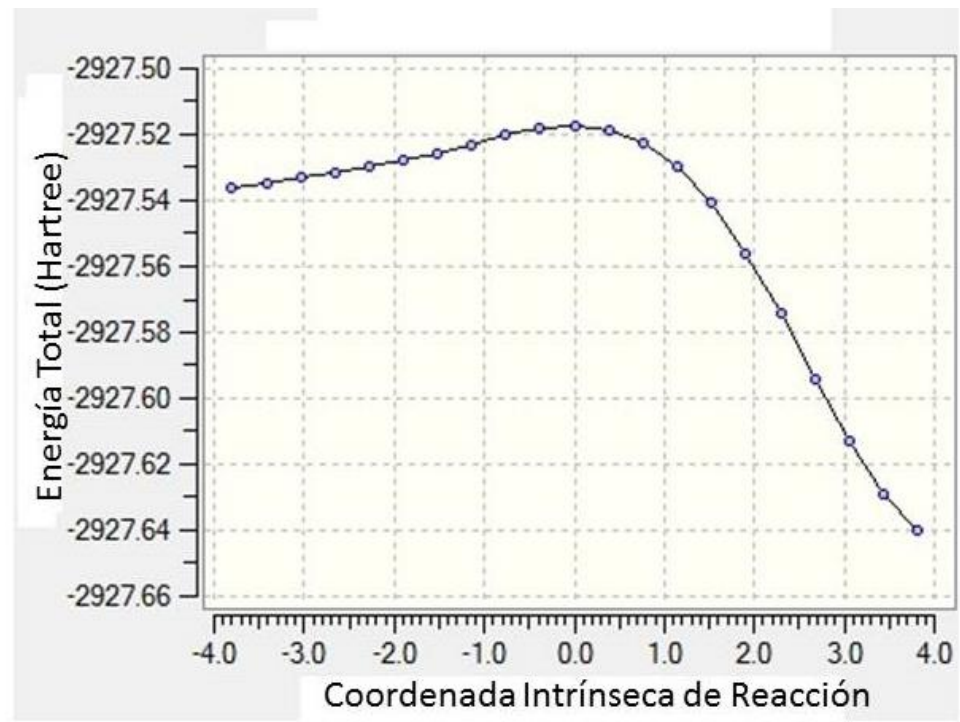

Figura 3: Energía Total a lo largo de la coordenada intrínseca de reacción 


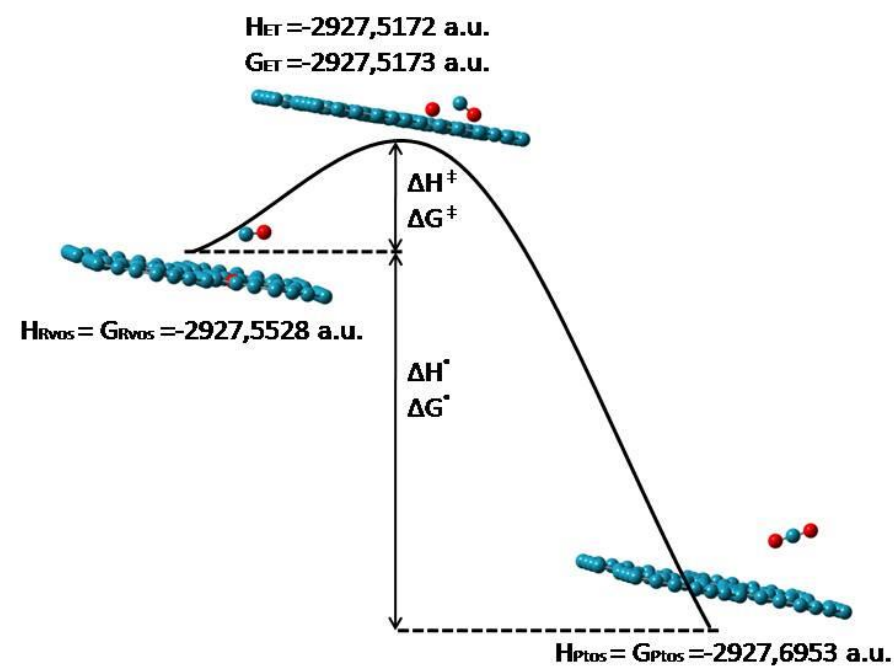

Figura 4: Diagrama de energías para la oxidación de CO sobre una lámina de $\beta$-grafino.

Tabla 3: Entalpia y energía libre de activación y de reacción y frecuencia para el mecanismo de oxidación.

\begin{tabular}{c|c|c|c|c}
\hline$\Delta \mathbf{H}^{\ddagger}[\mathbf{k c a l} / \mathbf{m o l}]$ & $\Delta \mathbf{G}^{\ddagger}[\mathrm{Kcal} / \mathbf{m o l}]$ & $\boldsymbol{\Delta} \mathbf{G}^{\circ}[\mathrm{Kcal} / \mathbf{m o l}]$ & $\boldsymbol{\Delta} \mathbf{G}[\mathrm{Kcal} / \mathbf{m o l}]$ & Frec $\left[\mathbf{c m}^{-1}\right]$ \\
\hline 22,34 & 22,28 & $-89,42$ & $-89,42$ & $604,16 \mathrm{i}$ \\
\hline
\end{tabular}

De acuerdo con los productos formados, se planteó un mecanismo en donde el proceso es concertado en una sola etapa. Además los valores negativos para la entalpía de reacción y energía libre de reacción [Tabla 3] indican que la reacción es exotérmica, exergónica y con la energía interna necesaria para que la reacción química se lleve a cabo en condiciones normales.

\section{CONCLUSIONES}

La coexistencia de carbonos con hibridación sp y sp2 en grafino promueve que átomos de carbono perciban características electropositivas. Esto puede mejorar notablemente la disociación de oxígeno promoviendo una mayor eficiencia de este como oxidante. La adsorción de oxígeno y monóxido de carbono y el análisis de las energías de adsorción nos permitió escoger el sistema GR/O-II como sistema de partida para la ruta de oxidación utilizando para ello el mecanismo Eley-Rideal. Los resultados obtenidos para los parámetros termodinámicos, indican que la reacción de oxidación a través del mecanismo Eley- Rideal es un proceso concertado, exotérmico y espontaneo, sugiriendo que este método es el más apropiado para esta reacción, sin embargo en futuros estudios se pretende abordar la oxidación de monóxido de carbono a partir del mecanismo de Langmuir-Hinshelwood.

\section{AGRADECIMIENTOS}

Al equipo de trabajo del Centro de Investigaciones Fisicoquímicas, Teóricas y Aplicadas de la Facultad de Ciencias Exactas y Naturales- Universidad Nacional de Catamarca

\section{BIBLIOGRAFÍA}

[1] KORDESH K., SIMADER G., Fuel Cells and their applications, 1 ed., , New York, VCH Publishers, 1996.

[2] YU, E.H., KREWER, U., SCOTT, K, "Principles and Materials Aspects of Direct Alkaline Alcohol Fuel Cells", Energies, vol. 3, n. 8, pp. 1499-1528, Ago. 2010. 
[3] LIU, H., LEE J.Y., "Electric Field Effects on the Adsorption of CO on a GrapheneNanodot and the Healing Mechanism of a Vacancy in a GrapheneNanodot", The Journal of Physical Chemistry C, v. 116, pp. 3034-3041, Ene. 2012

[4] PAN, D., OMBABA, M., ZHOU, Z.Y., et al., "Direct growth of carbon nanofibers to generate a 3D porous platform on a metal contact to enable an oxygen reduction reaction", ACS Nano, v. 6, n.12 pp. 10720 10726, Nov. 2012.

[5] ZHAO, Y., YANG, L., CHEN, S., et al., "Can boron and nitrogen co-doping improve oxygen reduction reaction activity of carbon nanotubes?", Journal of the American Chemical Society, v. 135, n. 4, pp. 12011204, Ene. 2013.

[6] GONG, K., DU, F., XIA, Z., et al., "Nitrogen-doped carbon nanotube arrays with high electrocatalytic activity for oxygen reduction”, Science, v. 323, pp. 760-764, Feb. 2009.

[7] YANG L., JIANG S., ZHAO Y., et al., "Boron-doped carbon nanotubes as metal-free electrocatalysts for the oxygen reduction reaction", Angewandte Chemie International Edition, v. 50, n. 31, pp. 7132-7135, Jun. 2011.

[8] WU, P., DU, P., ZHANG, H., et al., "GraphyneAs a Promising Metal-Free Electrocatalyst for Oxygen Reduction Reactions in Acidic Fuel Cells: A DFT Study", The Journal of Physical Chemistry C, v. 116, pp. 20472-20479, Sep. 2012.

[9] BAUGHMAN R.H., ECKHARDT H., KERTESZ M.J, "Structure-property predictions for new planar forms of carbon: Layered phases containing $\mathrm{sp}^{2}$ and $\mathrm{sp}$ atoms", The Journal of ChemicalPhysics, v. 87, n.11,pp. 6687-6699, Dic. 1987.

[10] GAUSSIAN 09, Revision A.1, FRISCH M. J. et al. Gaussian, Inc.,Wallingford CT, 2009.

[11] DENNINGTO R., KEITH T., MILLAM J., Semichem Inc., Shawnee Mission, KS, 2009

[12] PERDEW J.P., "Unified Theory of Exchange and Correlation Beyond the Local Density Approximation”, In: Electronic Structure of Solids '91, ZIESCHE P., ESCHIG H., AkademieVerlag, pp.11-20, 1991.

[13] BURKE, K., PERDEWJ, P., WANG, Y. "Derivation of a Generalized Gradient Approximation: the PW91 Density Functional", In: Electronic Density Functional Theory: Recent Progress and New Directions, ed. DOBSONJ. F., VIGNALE G., DAS, M.P., Ed. Plenum, pp.81-111, 1998.

[14] HEREDIA, C.L., FERRARESI-CUROTTO, V., LÓPEZ, M.B., "Characterization of Ptn (n = 2-12) clusters through global reactivity descriptors and vibrational spectroscopy, a theoretical study", Computational Materials Science, v.53, n.1, pp 18-24, Oct. 2011.

[15] ENGEL T. and ERTL G., "Elementary Steps in the Catalytic Oxidation of Carbon Monoxide on Platinum Metals", Advance in Catalysis, v. 28, Academic Press, pp.1-78, 1979

[16] RINCÓN, E. Y., TORO-LABBÉ, A., "Reaction force and electron localization function analysis of the metal chelation process in Mg(II)-thymine complex", Chemical Physics Letters, v.438, n. 1-3, pp93-98, Feb. 2007.

[17] CONTRERAS J.G., GERLI L.A., "Conformational Preference in 4,6-Dimethyl-1,3-Thioxane", Journal of the Chilean Chemical Society, v. 53, n. 1, pp.1389-1392, Mar. 2008.

[18] MOHAJERI, A., JENABI, M.J. "Singlet and triplet potential energy surfaces of C3H2", Journal of Molecular Structure: THEOCHEM, v. 820, pp.65-73, Jun. 2007 